\title{
P 200 THE EVOLUTION OF CARING FOR DYING PATIENTS IN INTENSIVE CARE UNITS IN THE UNITED STATES FROM 1960-1980
}

Waraporn Kongsuwan, ${ }^{1}$ Kathryn Keller, ${ }^{2}$ Susan Chase ${ }^{3} .{ }^{1}$ Prince of Songkla University, Hat-Yai, Songkhla, Thailand; ${ }^{2}$ Florida Atlantic University, Boca Raton, Florida, USA; ${ }^{3}$ University of Central Florida, Orando, Florida, USA

10.1136/bmjspcare-2014-000654.241

Introduction Since Intensive Care Units (ICUs) appeared in the United States in the late 1950's, nurses working in them have always cared for the dying patients. A historical reflection of the evolution of caring for dying patients in ICUs would assist in care model development. To discover retrospectively how and why caring for dying patients in the ICUs has changed can support ICU nurses in the present in developing their future care of the dying.

Purpose To examine the evolution of caring for dying patients in intensive care units in the United States from 1960-1980.

Methods Historical research method was used in this study. Data were obtained from primary and secondary sources, including electronic databases, critical care journals, and text books. Three intensive care nurses who practiced within the timeframe participated in the oral history interviews. The data were analyzed by using content analysis. Validity and reliability were established.

Findings The three major themes that emerged were: lack of formal preparation for care of the dying (1960-1980); increased workload and stress (1970-1980); and becoming more concerned about quality of end-of-life care (mid 1970s-1980).

Conclusion Nurses and healthcare professionals in the United States and worldwide could use this knowledge as a source to compare and develop their future practices in care for dying patients in the intensive care units. 